\title{
Effect of Nonrandom Mixing of Molecules and Holes on Phase Diagram in Mixture
}

\author{
Mamoru OKADA and Takuhei NosE \\ Department of Polymer Chemistry, Tokyo Institute of Technology, \\ Ookayama, Meguro-ku, Tokyo 152, Japan.
}

(Received December 24, 1980)

\begin{abstract}
The effect of nonrandom mixing of molecules and holes on phase diagrams of liquid mixtures has been investigated by comparing the numerical results calculated from the first and zeroth approximations of the hole theory. To study the effect of nonrandom mixing of molecules, calculations have also been made for the case in which the hole is not considered. In general, the nonrandom distribution of holes has a larger effect on phase diagrams than that of molecules. Nonrandomness increases or decreases compatibility depending on the system considered. The pressure dependence of compatibility has been also calculated using the first and zeroth approximations.
\end{abstract}

KEY WORDS Nonrandomness / Phase Diagram / Hole Theory / Polymer Solution / Pressure /

The lower critical solution temperature (LCST) is known to be general for nonpolar polymer solutions rather than the exception. ${ }^{1}$ The LCST is caused by a sort of condensation of a more volatile component. ${ }^{2}$ The free volume theories, presented by Prigogine, ${ }^{3,4}$ Flory, ${ }^{5-7}$ and their coworkers, can predict the LCST and can explain other thermodynamic behavior qualitatively. Therefore, it is apparent that free volume plays an important role in the thermodynamics of polymer solutions. In these theories, the same free volume is assigned to each molecule. In a real fluid mixture, however, the average size of vacant space around a molecule as well as the distribution of molecules of different species around it, should depend on the cohesive energy of the molecule considered. Renuncio and Prausnitz ${ }^{8}$ have suggested that one limitation of the Flory theory might be the random-mixing assumption for component molecules. The nonrandom distribution of vacant space might also have appreciable influence on solution properties.

Recently, Sanchez and Lacombe ${ }^{9-11}$ presented a simple type of hole theory called the lattice fluid theory, which allows for the volume change of a system and predicts the LCST. In this theory, the cell volume is fixed and the hole plays the same role as the free volume. It is reasonable to assume that the nonrandom distribution of vacant space can be represented by the nonrandom mixing of holes and molecules in the hole theory with fixed cell volume. In previous papers, ${ }^{12,13}$ we applied the quasichemical treatment to the lattice fluid theory from this viewpoint and investigated the effects of nonrandom mixing of holes and molecules on various thermodynamic quantities. The present paper investigates the effect of nonrandom mixing on phase diagrams which are very sensitive to the parameters characterizing the system. Another purpose of this paper is to study various features of phase diagrams predicted by the hole theory. This theory can be applied to gas-liquid equilibrium, but here we limit ourselves to investigating liquid-liquid phase diagrams.

\section{THEORETICAL}

As regards the calculation method, the lattice fluid theory is very similar to the Flory-Huggins theory. The main difference lies only in the fact that an additional component, i.e., the hole, is created or destroyed depending on thermodynamic conditions. Thus, we can apply the quasi-chemical treatment (referred to as the first approximation) to the lattice fluid theory. In the quasi-chemical treatment, ${ }^{14,15}$ 
the partition function is obtained by considering the partition of contact pairs instead of the partition of the molecules themselves. The number of contact pairs depends on the coordination number $z$ of the lattice, and the quasi-chemical treatment reduces to the lattice fluid theory at the limit of infinite $z$. Here, we introduce another approximation referred to as the zeroth approximation which assumes a random mixing of components on a lattice of a finite coordination number. The effect of nonrandom mixing on various quantities can be found by comparing the numerical results calculated from the first approximation with those calculated from the zeroth approximation for the same coordination number.

We presented below the thermodynamic functions necessary for calculating only phase diagrams. A detailed description of the theory is presented in ref 12 and 13 . We consider a binary system which consists of $N_{1} r_{1}$-mers, $N_{2} r_{2}$-mers and $N_{0}$ holes on a lattice of coordination number $z$. The lattice deformation in the mixing process is ignored; that is, $z$ and the volume of a lattice site $v^{*}$ are assumed to be independent of composition. The following notations are introduced to simplify expression:

$$
\begin{aligned}
& q_{i}=r_{i}(z-2)+2 \quad(i=1,2) \\
& N_{r}=N_{0}+r_{1} N_{1}+r_{2} N_{2} \\
& N_{q}=N_{0}+q_{1} N_{1}+q_{2} N_{2} \\
& u_{0}=N_{0} / N_{q} \\
& u_{i}=q_{i} N_{i} / N_{q} \quad(i=1,2)
\end{aligned}
$$

The chemical potential $\mu_{1}$ of component 1 at temperature $T$ and pressure $P$ is calculated in the first approximation as,

$$
\begin{aligned}
\mu_{1}= & -q_{1} \varepsilon_{11}^{*}-k T\left\{\ln \left(\delta_{1} / \sigma_{1}\right)-\ln N_{1}\right. \\
& -(z / 2-1) r_{1} \ln N_{r}+(z / 2) q_{1} \ln N_{q} \\
& \left.-(z / 2) q_{1} \ln \left[\left(u_{1}-y_{10}-y_{12}\right) / u_{1}^{2}\right]\right\}+P r_{1} v^{*}
\end{aligned}
$$

where $\delta_{1}$ and $\sigma_{1}$ are a flexibility parameter and the symmetry number of component 1 , respectively, and $k$ is the Boltzmann constant. The parameter $\delta_{i}$ is defined as the number of ways in which an $r_{i}$-mer can be arranged after one of its end segments has been placed on a lattice site. The quantities $y_{i j}$ $(i, j=0,1,2)$ which correspond to the number of contacts between components $i$ and $j$ (the subscript 0 denotes a hole) are determined by the following equations:

$$
\begin{gathered}
\left(u_{0}-y_{01}-y_{02}\right)\left(u_{1}-y_{01}-y_{12}\right) / y_{01}^{2} \\
=\exp \left(2 \varepsilon_{11}^{*} / z k T\right) \\
\left(u_{0}-y_{01}-y_{02}\right)\left(u_{2}-y_{02}-y_{12}\right) / y_{02}^{2} \\
=\exp \left(2 \varepsilon_{22}^{*} / z k T\right) \\
\left(u_{1}-y_{01}-y_{12}\right)\left(u_{2}-y_{02}-y_{12}\right) / y_{12}^{2} \\
=\exp \left[2\left(\varepsilon_{11}^{*}+\varepsilon_{22}^{*}-2 \varepsilon_{12}^{*}\right) / z k T\right]
\end{gathered}
$$

where $-2 \varepsilon_{i j}^{*} / z$ denotes the contact energy between the segments of component $i$ and $j$. The contact energy $\varepsilon_{12}^{*}$ is expressed in the following form:

$$
\varepsilon_{12}^{*}=\zeta\left(\varepsilon_{11}^{*} \varepsilon_{22}^{*}\right)^{1 / 2}
$$

The parameter $\zeta$ measures the deviation from the geometrical-mean rule (the Berthelot rule) and usually has a value slightly less than unity for a nonpolar mixture. ${ }^{16}$ The value of $N_{0}$ or the volume $V$ of the system is determined by the equation of state:

$$
\begin{aligned}
P v^{*} / k T= & (z / 2-1) \ln (1-1 / \tilde{V}) \\
& -(z / 2) \ln \left(1-y_{01} / u_{0}-y_{02} / u_{0}\right)
\end{aligned}
$$

where $\tilde{V}$ is the reduced volume defined by

$$
\tilde{V}=V / V^{*}, \quad V^{*}=\left(r_{1} N_{1}+r_{2} N_{2}\right) v^{*}
$$

Corresponding expressions calculated from the zeroth approximation are as follows: For the chemical potential,

$$
\begin{aligned}
\mu_{1}= & -q_{1} \varepsilon_{11}^{*}+q_{1}\left[\left(u_{1}-1\right)^{2} \varepsilon_{11}^{*}+u_{2}^{2} \varepsilon_{22}^{*}\right. \\
& \left.+2\left(u_{1}-1\right) u_{2} \varepsilon_{12}^{*}\right]-k T\left[\ln \left(\delta_{1} / \sigma_{1}\right)-\ln N_{1}\right. \\
& \left.-(z / 2-1) r_{1} \ln N_{r}+(z / 2) q_{1} \ln N_{q}\right] \\
& +P r_{1} v^{*}
\end{aligned}
$$

and for the equation of state,

$$
\begin{aligned}
P v^{*} / k T= & (z / 2-1) \ln (1-1 / \tilde{V}) \\
& -(z / 2) \ln u_{0}-\left(u_{1}{ }^{2} \varepsilon_{11}^{*}+u_{2}{ }^{2} \varepsilon_{22}^{*}\right. \\
& \left.+2 u_{1} u_{2} \varepsilon_{12}^{*}\right) / k T
\end{aligned}
$$

Coexistence curves are determined by the equilibrium conditions:

$$
\begin{aligned}
& \mu_{1}\left(P, T, \phi_{1}{ }^{\prime}\right)=\mu_{1}\left(P, T, \phi_{1}{ }^{\prime \prime}\right) \\
& \mu_{2}\left(P, T, \phi_{2}{ }^{\prime}\right)=\mu_{2}\left(P, T, \phi_{2}{ }^{\prime \prime}\right)
\end{aligned}
$$

where the prime and double prime denote the two 
phases at equilibrium, and $\phi_{i}$ is the segment fraction defined by

$$
\phi_{i}=r_{i} N_{i} /\left(r_{1} N_{1}+r_{2} N_{2}\right) \quad(i=1,2)
$$

We also calculate the coexistence curves using the original Guggenheim's treatment, where no hole exists, in order to make comparison with the present treatment. According to Guggenheim's treatment, ${ }^{14,15}$ the chemical potential is expressed to the first approximation by

$$
\begin{aligned}
\mu_{1}= & -q_{1} \varepsilon_{11}^{*}-k T\left\{\ln \left(\delta_{1} / \sigma_{1}\right)-\ln N_{1}\right. \\
& -(z / 2-1) r_{1} \ln N_{r}+(z / 2) q_{1} \ln N_{q} \\
& \left.-(z / 2) q_{1} \ln \left[\left(u_{1}-y\right) / u_{1}^{2}\right]\right\}
\end{aligned}
$$

where

$$
y=2 u_{1} u_{2} /(\beta+1)
$$

and

$$
\begin{aligned}
\beta^{2}=1 & +4 u_{1} u_{2}\left\{\operatorname { e x p } \left[2 \left(\varepsilon_{11}^{*}+\varepsilon_{22}^{*}\right.\right.\right. \\
& \left.\left.\left.-2 \varepsilon_{12}^{*}\right) / z k T\right]-1\right\}
\end{aligned}
$$

and to the zeroth approximation, $\mu_{1}$ is given by

$$
\begin{aligned}
\mu_{1}= & -q_{1} \varepsilon_{11}^{*}+q_{1} u_{2}^{2}\left(\varepsilon_{11}^{*}+\varepsilon_{22}^{*}-2 \varepsilon_{12}^{*}\right) \\
& -k T\left[\ln \left(\delta_{1} / \sigma_{1}\right)-\ln N_{1}\right. \\
& \left.-(z / 2-1) r_{1} \ln N_{r}+(z / 2) q_{1} \ln N_{q}\right]
\end{aligned}
$$

In eq 15-17, the quantities $N_{r}, N_{q}$ and $u_{i}$ are obtained from eq 2, 3 and 5 by setting $N_{0}=0$.

\section{NUMERICAL RESULTS AND DISCUSSION}

The parameters of the present theory are considered to be of the same order as those of the lattice fluid theory. ${ }^{12}$ The values of the parameters used in the following calculations were determined from those of the lattice fluid theory which are tabulated for various substances by Sanchez and Lacombe. ${ }^{10,11}$ We set $v^{*}\left(=v_{1}{ }^{*}=v_{2}{ }^{*}\right)=1 \times 10^{-5}$ $\mathrm{m}^{3} \mathrm{~mol}^{-1}$ and $z=12$.

\section{Effect of Nonrandomness on Phase Diagram}

Figure 1 shows the coexistence curves at $P=0.1$ MPa for the components 1 and 2 which have the same chain length but have largely different contact energies or characteristic temperatures: $r_{1}=r_{2}=10$, $T_{1}{ }^{*} \equiv \varepsilon_{11}^{*} / k=500 \mathrm{~K}$, and $T_{2}{ }^{*} \equiv \varepsilon_{22}^{*} / k=700 \mathrm{~K}$. For the parameter $\zeta$, to which phase diagrams are very sensitive, we set $\zeta=0.95$ so as to have the critical point appear in an appropriate temperature range. In the case where no hole exists, the coexistence curve calculated from the first approximation (1st) appears at lower temperatures than that calculated from the zeroth approximation (0th). Thus, the compatibility is lowered when the nonrandom mix-

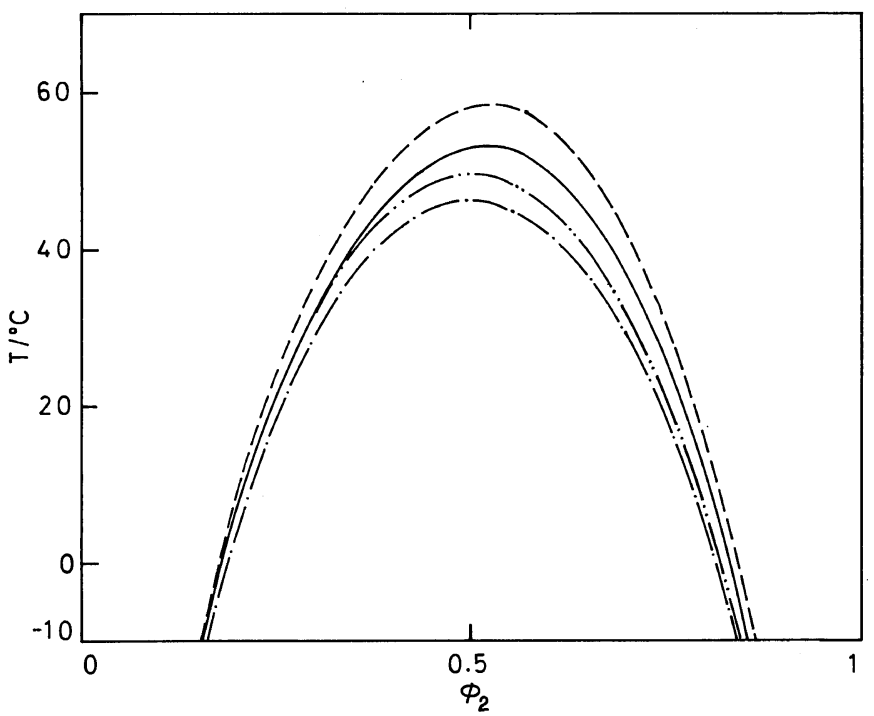

Figure 1. Liquid-liquid phase diagram calculated from various approximations to illustrate the effect of nonrandomness in a system of low molecular weight molecules. $P=0.1 \mathrm{MPa}$. Each component is characterized as $r_{1}=r_{2}=10, T_{1}{ }^{*}=500 \mathrm{~K}, T_{2}{ }^{*}=700 \mathrm{~K}$, and $\zeta=0.95:-, 1$ st approximation;,--- 0 th approximation; --.---, 1st approximation without a hole; $-\cdots-\cdots-, 0$ th approximation without a hole. 


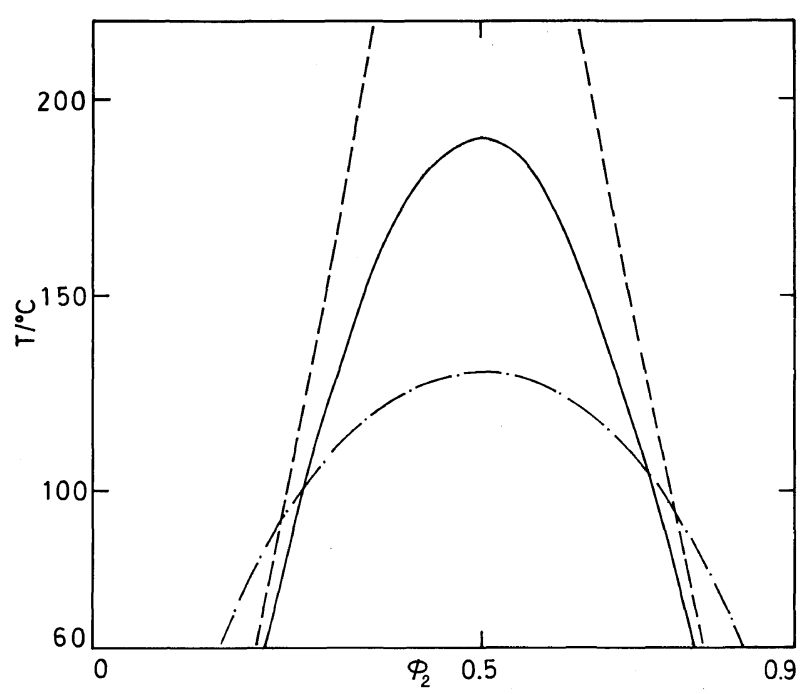

Figure 2. Phase diagram. Effect of nonrandomness in a system of large molecules. $r_{1}=r_{2}=1000$, $T_{1}{ }^{*}=500 \mathrm{~K}, T_{2}{ }^{*}=535 \mathrm{~K}$, and $\zeta=1$ except that $T_{2}{ }^{*}=545 \mathrm{~K}$ for the case without a hole:,- 1 st; - - , 0 th; $-\cdot-\cdot-$, lst and 0 th without a hole.

ing of component molecules is taken into account. This result is very reasonable since the nonrandom mixing of molecules suppresses the contribution from the contact-energy difference that gives rise to phase separation in the present case.

In the treatment with holes, the relation between the 0 th and 1 st approximations remains unchanged: nonrandomness increases compatibility and the distance between the curves of the 0th and 1st (broken curve and solid curve in Figure 1) is about the same as that of the treatment without holes. Therefore, the nonrandom distribution of holes does not play an important role in this case. The existence of holes expands the incompatible region. This effect is related to the free-volume dissimilarity between the two components. It is seen from Figure 1 that, in the system of components with the same chain length, the phase diagram is symmetric in the treatment without holes, while it is asymmetric in the treatment with holes.

As the chain length increases, the combinatorial entropy decreases and small difference in contact energy can give rise to phase separation. Thus, the contributions other than combinatorial entropy become more effective. Figure 2 illustrates such a case, where $r_{1}=r_{2}=1000, T_{1}{ }^{*}=500 \mathrm{~K}, T_{2}{ }^{*}=535 \mathrm{~K}$, and $\zeta=1$. Since the critical temperature $T_{\mathrm{c}}$ is very low in the treatment without holes, the coexistence curve for $T_{2}{ }^{*}=545 \mathrm{~K}$ is shown in the figure. When no hole exists, the curves calculated from the 0 th and 1st are almost indistinguishable on the scale of this figure because of the small energy difference. In contrast to this, the calculations with holes show a large contribution of nonrandomness to the phase diagram in spite of a smaller energy difference. In this system, nonrandomness increases compatibility as in the system treated above (Figure 1), but its effect is more remarkable.

In general, free volume plays a very important role in mixtures of components with largely different chain lengths. Figure 3 shows the coexistence curves, calculated from various treatments, for the system of components characterized by $r_{1}=10$, $r_{2}=300, T_{1}{ }^{*}=500 \mathrm{~K}, T_{2}{ }^{*}=600 \mathrm{~K}$, and $\zeta=0.98$. The hole theory predicts the lower critical solution temperature (LCST), which is general for polymer solutions, as well as the upper critical solution temperature (UCST). In the system without holes, the curves calculated from the 0 th and 1st are almost indistinguishable in the figure, which means that the contribution from the contact-energy difference may be considered comparatively small.

In the system with holes, the compatibility decreases when nonrandomness is taken into account, even for the UCST, which is different from the preceding systems $\left(r_{1}=r_{2}\right)$. The phase separation 


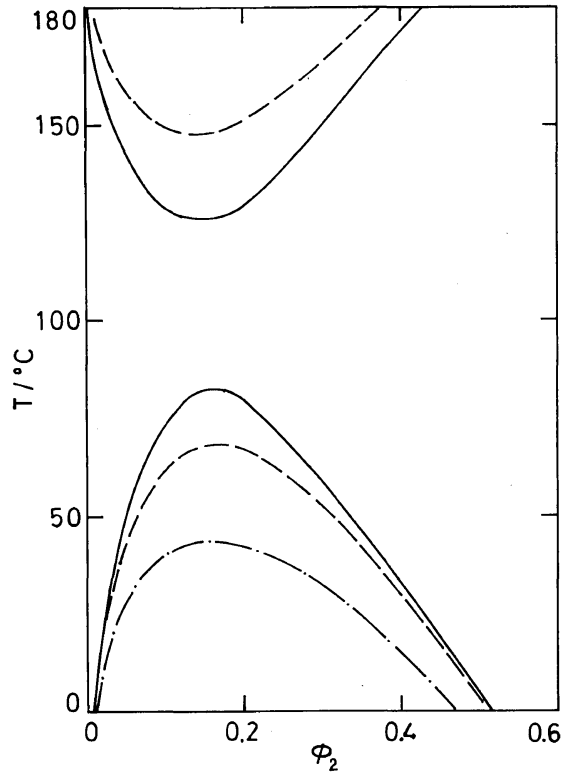

Figure 3. Phase diagram. Effect of nonrandomness in a system of molecules largely differing in size. $r_{1}=10$, $r_{2}=300, T_{1}{ }^{*}=500 \mathrm{~K}, T_{2}{ }^{*}=600 \mathrm{~K}$, and $\zeta=0.98$. Each curve denotes the same approximation as in Figure 2.

with an UCST (i.e., phase separation which occurs when the temperature is lowered) is caused by a contact-energy dissimilarity, and the incompatible region is also extended by the free-volume difference between the pure components. Therefore, we have to take into consideration the effect of the nonrandom mixing of molecules and holes on the free volumes of the pure components even for the UCST. It has been shown in a previous paper ${ }^{12}$ that the nonrandom distribution of holes gives rise to an expansion of volumes of the pure components, which leads to a larger difference in free volume. The free-volume dissimilarity is known to decrease compatibility. In this system, this effect is larger than the effect of nonrandomness' suppressing the contribution from the difference in contact energy between the components. In fact, as mentioned above, nonrandomness has virtually no effect on the phase diagram of this system when no hole exists.

Compatibility is decreased by nonrandomness at both the UCST and the LCST in this system. The extension of the incompatible region by nonrandomness at the LCST is larger than that at the UCST. This is reasonable because the LCST is caused by the free-volume difference between the

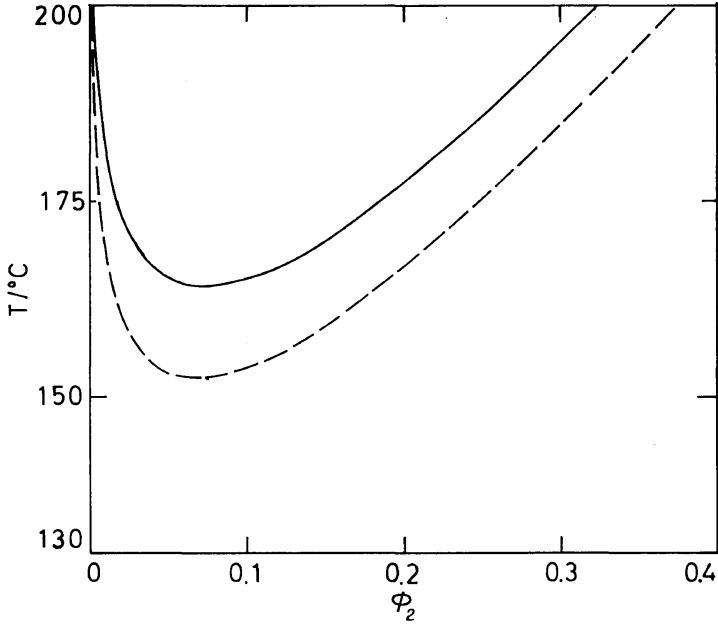

Figure 4. Phase diagram. Effect of nonrandomness in a system of large molecules having a strong interaction. $r_{1}=900, \quad r_{2}=1000, \quad T_{1}{ }^{*}=500 \mathrm{~K}, \quad T_{2}{ }^{*}=700 \mathrm{~K}, \quad$ and $\zeta=1.04$ :,$- 1 \mathrm{st} ;---, 0$ th.

components, which is increased by nonrandomness as mentioned above.

At the LCST, as well as at the UCST, the 1st approximation does not always predict poorer compatibility than the 0th approximation. An example of this is shown in Figure 4. The components are long chains $\left(r_{1}=900, r_{2}=1000\right)$ having different characteristic temperatures $\left(T_{1}^{*}=500 \mathrm{~K}, T_{2}{ }^{*}=\right.$ $700 \mathrm{~K})$ and a strong interaction $(\zeta=1.04)$. We have selected this combination of components as a model system for a partially compatible polymerpolymer pair, which is usually exothermic. ${ }^{17}$ At low temperatures, the strong interaction $(\zeta>1)$ causes the compatibility throughout the entire concentration range, but as the temperature is raised, the mixture separates into two phases. An asymmetric phase diagram is obtained in spite of nearly the same chain lengths of the components. Possible causes for an increase in compatibility owing to nonrandomness near the LCST are as follows. Since the contact between different species is more likely to occur than that between the same species in a case where $\varepsilon_{11}^{*}+\varepsilon_{22}^{*}<2 \varepsilon_{12}^{*}$, the number of contacts between different species is increased by nonrandom mixing of molecules, and this enhances the compatibility. In addition, we found, as indicated in a previous paper, ${ }^{13}$ that deviation from the randommixing value of the average number of holes in contact with component 2 is larger than that in 
contact with component 1 when $T_{2}{ }^{*}$ is larger than $T_{1}{ }^{*}$. In other words, the component which is in contact with more holes in a pure liquid than the other component is also in contact with more holes than the other in a mixture. This similarity in the hole distribution between a mixture and pure liquids probably reduces the difference in the entropy of mixing of holes and molecules between the mixture and liquids. Consequently, the negative value of entropy of mixing near the LCST, i.e., the excess entropy arising from the free-volume difference, will be small compared with the random mixing case. A smaller negative entropy results in better compatibility at the LCST.

\section{Comparison between the Zeroth Approximation and Lattice Fluid Theory}

Both the lattice fluid theory (LF) and the 0th approximation assume the random mixing of components, but in the 0th we give a finite value to the coordination number, but not in LF. The surface area of a chain molecule is decreased by the connectivity of the segments and depends on the coordination number $z$. The effect of connectivity diminishes with increasing $z$ and vanishes at the limit of infinite $z$. Therefore, even for molecules characterized by the same $T^{*}$, the total contact energy per molecule in the 0th is smaller than in LF. Such a situation is illustrated by the coexistence curves in Figures 5 and 6 . Figure 5 shows the same system as in Figure 1, and Figure 6 shows the same system as in Figure 3. The curve LFa are the coexistence curves calculated from LF with the same $T^{*}$ as in the 0th. Because of the higher cohesive energy resulting from a larger surface area, the coexistence curves LFa locate at much higher temperatures than those of the 0th

By multiplying $T^{*}$ of LF by $q / r$, we can correct the difference in total contact energy in a closepacked state arising from the difference in surface area. The curves $\mathrm{LFb}$ in Figures 5 and 6 indicate the coexistence curves obtained after this correction. The difference in the phase diagrams between LF and the 0 th becomes small as a result of correction for surface area, but the two diagrams do not coincide with each other, as shown by the figures. When $T^{*}$ decreases, the volume of a system at a fixed temperature becomes large and accordingly the difference in free volume becomes large, leading to poorer compatibility. Thus, in Figure 6, the

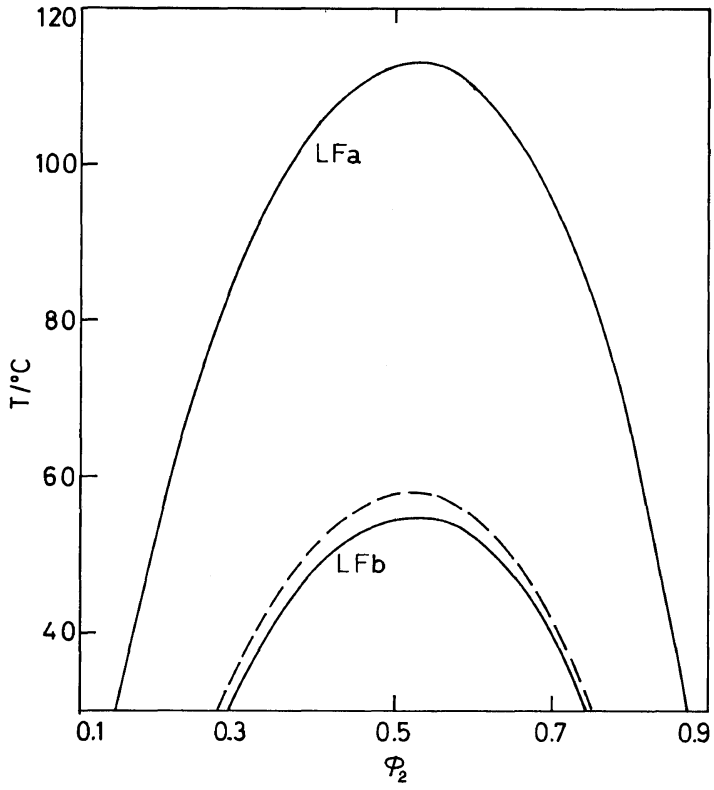

Figure 5. Phase diagram. Comparison between the 0th and lattice fluid theory (LF). $r_{1}=r_{2}=10, \zeta=0.95$ : $T_{1}{ }^{*}=500 \mathrm{~K}, T_{2}{ }^{*}=700 \mathrm{~K}\left(0\right.$ th and LFa); $T_{1}{ }^{*}=425 \mathrm{~K}$, $T_{2}{ }^{*}=595 \mathrm{~K}(\mathrm{LFb}):-, \mathrm{LF} ;---, 0$ th.

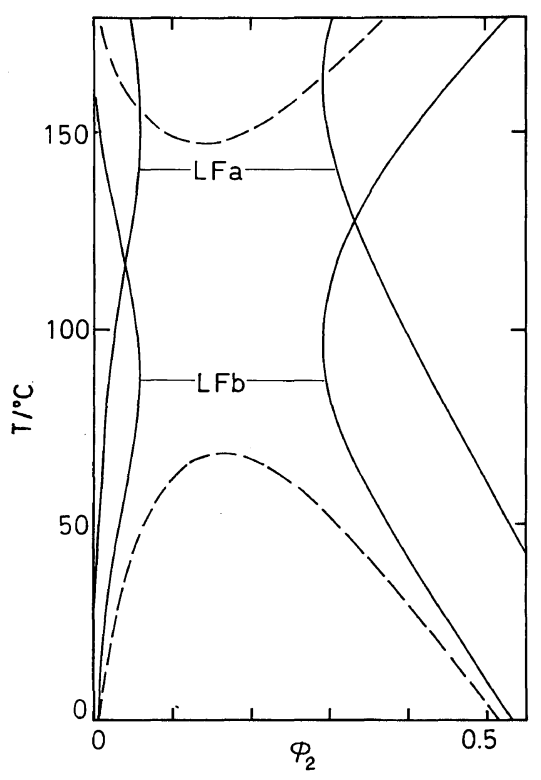

Figure 6. Phase diagram. Comparison between the 0th and lattice fluid theory. $r_{1}=10, r_{2}=300, \zeta=0.98$ : $T_{1}{ }^{*}=500 \mathrm{~K}, T_{2}{ }^{*}=600 \mathrm{~K}(0 \mathrm{th}$ and $\mathrm{LFa}) ; T_{1}{ }^{*}=425 \mathrm{~K}$, $T_{2}{ }^{*}=510 \mathrm{~K}(\mathrm{LFb})$. Each curve denotes the same approximation as in Figure 5. 


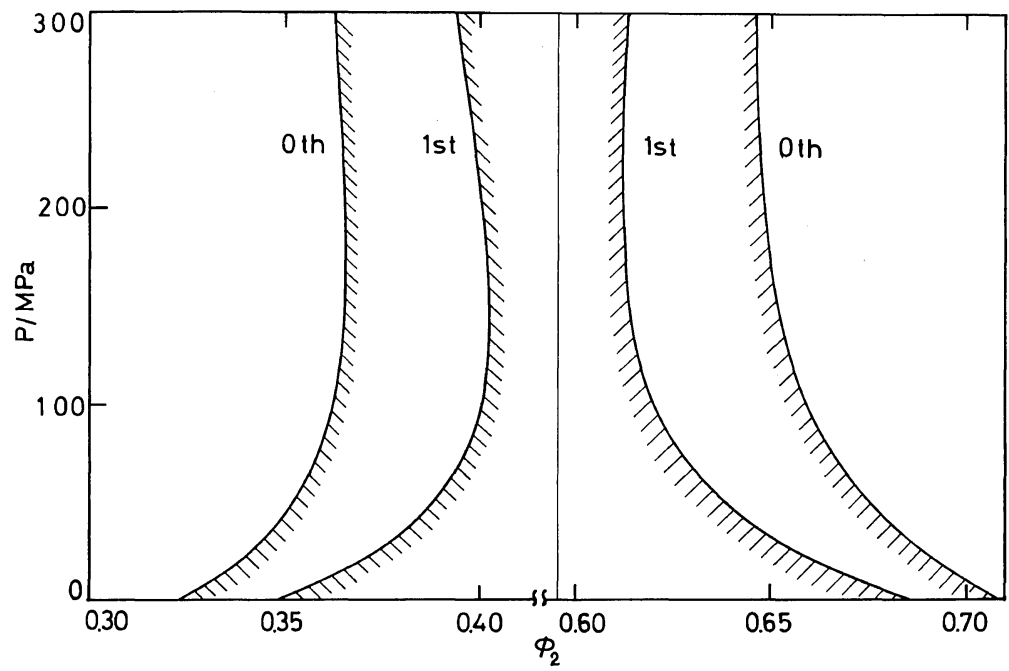

Figure 7. Pressure dependence of coexistence compositions at $40^{\circ} \mathrm{C}$ for the system in Figure 1 .

difference in the phase diagrams between the 0th and $\mathrm{LFb}$ arises from the difference in the reduced volume between them. However, in Figure 5 where the contribution from the free-volume difference to phase separation is small, the difference between the 0th and LFb may arise from an overestimation of the number of configurations of polymer chains in LF, since the theory assumes an infinite coordination number. In both Figures 5 and 6 , the difference in the phase diagrams between the 0 th and LF is larger than that between the 0th and 1st for the same $T^{*}$.

\section{Pressure Dependence}

Figures 7 and 8 show the temperature dependence of the coexistence compositions. In these figures, the two curves calculated from the 0th and 1 st show qualitatively the same pressure dependence. Figure 7 shows the pressure dependence at $T=40^{\circ} \mathrm{C}$ for the system in Figure 1. The compatibility increases with increasing pressure at low pressure, but at high pressure, it decreases slightly with increasing pressure. This implies that there exists a minimum in the pressure dependence of the critical temperature $T_{\mathrm{c}}$. The minimum in $T_{\mathrm{c}} v s . P$ for the UCST has been observed experimentally in several oligomeroligomer ${ }^{18,19}$ and polymer-solvent ${ }^{20}$ systems. At atmospheric pressure, the compatibility is decreased by the difference in free volume even for the UCST as mentioned before. Thus, as pressure is elevated, the compatibility should increase in the system in

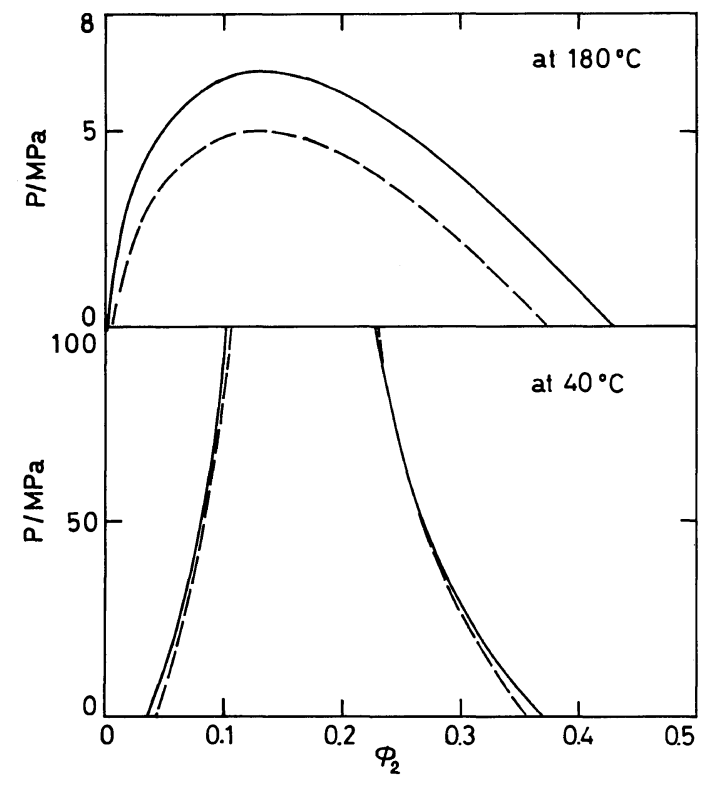

Figure 8. Pressure dependence of coexistence compositions at 40 and $180^{\circ} \mathrm{C}$ for the system in Figure 3: 1 st; - - , 0th.

which the free-volume difference is comparatively large, since the free-volume difference becomes smaller. Saeki et al. ${ }^{21}$ investigated the pressure dependence of the UCST in the polystyrenecyclohexane system in which the polymer had various molecular weights. According to their measurements, $(d T / d P)_{\mathrm{c}}$ is negative at low pressure 
for high molecular weight polystyrene, increases with decreasing molecular weight and changes its sign from negative to positive. This also indicates that the negative value of $(d T / d P)_{\mathrm{c}}$ at low pressure is caused by free-volume difference, since this difference increases with increasing molecular weight of polystyrene.

Figure 8 shows the pressure dependence of the coexistence compositions at 40 (below the UCST at $P=0.1 \mathrm{MPa}$ ) and $180^{\circ} \mathrm{C}$ (above the LCST at $P=0.1$ $\mathrm{MPa}$ ) for the system shown in Figure 3. At $180^{\circ} \mathrm{C}$, compatibility is attained in the whole concentration range by elevating the pressure above a certain value. The pressure dependence of the LCST is larger than that of the UCST in accordance with experimental observation in a polymer solution. ${ }^{22}$ The pressure dependence of the compatibility at $40^{\circ} \mathrm{C}$ is similar to the preceding case (Figure 7), but the pressure has greater influence on the compatibility in the present case than in the preceding case because of the larger difference in free volume.

\section{CONCLUSION}

The effect of nonrandomness on liquid-liquid coexistence curves was investigated by comparing the numerical results calculated from the first and zeroth approximations of the hole theory. The original Guggenheim's treatment, where a hole is not considered, was also used in the calculations to separate the effect of the nonrandom distribution of holes from the effect of the nonrandom mixing of component molecules.

The main results are as follows: Generally, the nonrandom distribution of holes has a much larger effect on phase diagrams than does the nonrandom mixing of molecules. When no hole exists, the nonrandom mixing of molecules increases the compatibility since nonrandomness suppresses the contribution from the contact-energy difference between molecules. When holes exist, nonrandomness increases or decreases the compatibility depending on the system under consideration; in a system where the contact-energy difference is large, nonrandomness increases the compatibility, while in a system where the free-volume difference is large, nonrandomness decreases the compatibility. Thus, it is concluded that nonrandomness has two different effects on the compatibility of a system. That is, the nonrandom mixing of holes and molecules expands the volume of a liquid and enhances the difference in free volume between components, and this in turn decreases compatibility. On the other hand, nonrandomness suppresses the contribution from the unpreferable contacts between different molecular species to incompatibility.

\section{REFERENCES}

1. P. T. Freeman and J. S. Rowlinson, Polymer, 1, 20 (1960).

2. D. Patterson, Macromolecules, 2, 672 (1969).

3. I. Prigogine, N. Trappeniers, and V. Mathot, Disc. Faraday Soc., 15, 93 (1953).

4. I. Prigogine, "The Molecular Theory of Solutions," North-Holland Publishers, Amsterdam, 1957.

5. P. J. Flory, R. A. Orwoll, and A. Vrij, J. Am. Chem. Soc., 86, 3507 (1964).

6. B. E. Eichinger and P. J. Flory, Trans. Faraday Soc., 64, 2035 (1968).

7. P. J. Flory, Disc. Faraday Soc., 49, 7 (1970).

8. J. A. R. Renuncio and J. M. Prausnitz, Macromolecules, 9, 898 (1976).

9. I. C. Sanchez and R. H. Lacombe, Nature (London), 252, 381 (1974).

10. I. C. Sanchez and R. H. Lacombe, J. Phys. Chem., 80, 2352, 2568 (1976).

11. I. C. Sanchez and R. H. Lacombe, Macromolecules, 11, 1145 (1978).

12. M. Okada and T. Nose, Polym. J., 13, 399 (1981).

13. M. Okada and T. Nose, Polym. J., 13, 591 (1981).

14. E. A. Guggenheim, Proc. R. Soc. London, Ser. A, 183, 203, 213 (1944).

15. E. A. Guggenheim, "Mixtures," Oxford University Press, London, 1952.

16. F. Kohler, Ber. Bunsenges. Phys. Chem., 81, 1037 (1977).

17. C. A. Cruz, J. W. Barlow, and D. R. Paul, Macromolecules, 12, 726 (1979).

18. B. A. Wolf and G. Blaum, Macromol. Chem., 180, 2591 (1979).

19. K. Hitachi, private communication: polystyrene $\left(M_{w}=2200\right)-n$-hexadecane.

20. H. Hosokawa, M. Nakata, T. Dobashi, and K. Kaneko, Rep. Prog. Polym. Phys. Jpn., 23, 13 (1980).

21. S. Saeki, N. Kuwahara, M. Nakata, and M. Kaneko, Polymer, 16, 445 (1975).

22. S. Saeki, N. Kuwahara, and M. Kaneko, Macromolecules, 9, 101 (1976). 